\title{
Antiproliferation activities of NK4 on multiple myeloma
}

\author{
WENZHONG QUE ${ }^{1-3}$, HUILI LIU ${ }^{4}$, QINQIN YANG ${ }^{5}$ and SHANGHUA XU ${ }^{6}$ \\ Departments of ${ }^{1}$ Rheumatology and ${ }^{2}$ Geriatric Medicine, The Affiliated Nanping First Hospital of Fujian Medical University, \\ Nanping, Fujian 353000; ${ }^{3}$ Department of Hematology and Rheumatology, The First Affiliated Hospital of Fujian Medical \\ University, Fuzhou, Fujian 350005; ${ }^{4}$ Department of Medical Technology, Zhang Zhou Health Vocational College, \\ Zhangzhou, Fujian 363000; ${ }^{5}$ College of Pharmacy, Fujian Medical University, Fuzhou, Fujian 350005; ${ }^{6}$ Department of \\ Cardiology, The Affiliated Nanping First Hospital of Fujian Medical University, Nanping, Fujian 353000, P.R. China
}

Received January 9, 2018; Accepted May 25, 2018

DOI: $10.3892 /$ etm.2018.6649

\begin{abstract}
Multiple myeloma (MM) is a plasma cell malignancy. The hepatocyte growth factor (HGF) has been demonstrated to promote MM cell growth. NK4, a splice variant of HGF in which the heavy chain consists of the N-terminal domain and the four kringle domains, is a specific antagonist of HGF that competes with HGF for tyrosine-protein kinase receptor binding. The current study aimed to examine the antiproliferative activity of NK4 on human MM cells and to investigate the underlying mechanism. The results indicated that NK4 suppressed proliferation and induced apoptosis in RPMI 8226 cells. In addition, NK4 altered the expression of cell cycle and apoptosis-associated proteins in RPMI 8226, including cyclin-dependent kinase 4, cyclin D1, cyclin-dependent kinase inhibitor $1 \mathrm{~B}$, apoptosis regulator Bcl-2, apoptosis regulator BAX, cleaved caspase-9 and caspase-3. Furthermore, NK4 inhibited the activation of the RAC- $\alpha$ serine/threonine-protein kinase (Akt)/serine/threonine-protein kinase mTOR (mTOR) signaling pathway and reduced the levels of phosphorylated (p)-Akt, p-mTOR, ribosomal protein S6 kinase beta-1 and eukaryotic initiation factor 4E binding protein 1 in RPMI 8226 cells. In conclusion, NK4 inhibited the proliferation of human MM RPMI 8226 cells, which may be attributed to the induction of apoptosis and the inhibition of the Akt/mTOR signaling pathway.
\end{abstract}

Correspondence to: Dr Wenzhong Que, Department of Rheumatology, The Affiliated Nanping First Hospital of Fujian Medical University, 317 Zhongshan Road, Nanping, Fujian 353000, P.R. China

E-mail: quewenzhong@163.com

Professor Shanghua Xu, Department of Cardiology, The Affiliated Nanping First Hospital of Fujian Medical University, 317 Zhongshan Road, Nanping, Fujian 353000, P.R. China

E-mail: xshanghua@163.com

Key words: hepatocyte growth factor antagonist NK4, multiple myeloma, RPMI 8226, proliferation, RAC- $\alpha$ serine/threonine-protein kinase/serine/threonine-protein kinase mTOR

\section{Introduction}

Multiple Myeloma (MM) is a plasma cell malignancy. To date, MM remains an incurable disease (1). Despite the development of novel drugs for MM, patients have a median survival of 5 years due to the resistance to these agents (1). Identifying novel targets for MM treatment is crucial. The hepatocyte growth factor (HGF) has been demonstrated to promote MM cell growth (2). Binding of HGF to the tyrosine-protein kinase (c-Met) receptor activates a signaling cascade, which regulates growth and survival of cancer cells (3). c-Met and its ligand HGF are overexpressed in the human MM cell lines JJN-3, U-266, OH-2, JW and primary human MM cells (4). Additionally, HGF/c-Met signaling promotes survival and drug resistance of MM cells in vitro and in vivo (5). Blocking of the HGF/c-Met signaling pathway inhibits MM cell proliferation $(6,7)$.

NK4, a splice variant of HGF in which the heavy chain consists of the $\mathrm{N}$-terminal domain and the four kringle domains, is a specific antagonist of HGF that competes with HGF for c-Met receptor binding to inhibit interactions between HGF and c-Met (8). For the current study, the RPMI 8226 MM cell line in which HGF and c-Met are highly expressed was chosen and adenovirus-mediated overexpression of NK4 was studied to examine antimyeloma effects of NK4 and to investigate underlying mechanisms.

\section{Materials and methods}

Cell culture and transduction. RPMI 8226 cells were purchased from American Type Culture Collection (Manassas, VA, USA) and cultured in RPMI 1640 medium (Gibco; Thermo Fisher Scientific, Inc., Waltham, MA, USA) supplemented with $10 \%$ fetal bovine serum (Gibco; Thermo Fisher Scientific, Inc.) at $37^{\circ} \mathrm{C}$ in a humidified atmosphere containing $5 \% \mathrm{CO}_{2}$. The construction of adenovirus (Ad) vectors Ad-NK4 and Ad-green fluorescent protein (GFP) was performed as described previously (9). Transduction of RPMI 8226 cells with Ad-NK4 or Ad-GFP (Ad-Control) was conducted according to a previously reported protocol (10). Cells were collected $48 \mathrm{~h}$ after transduction for further analysis. 

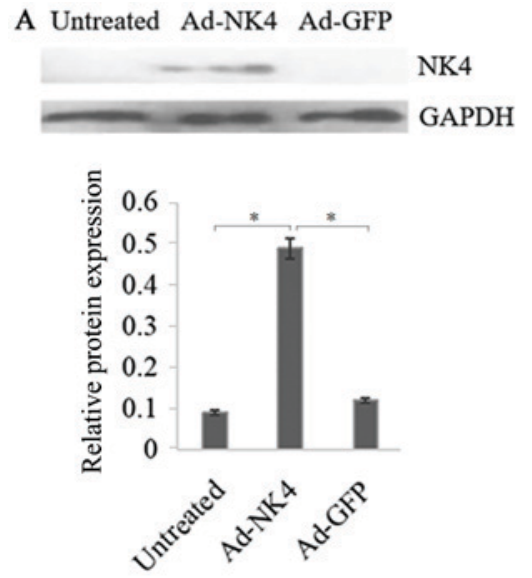
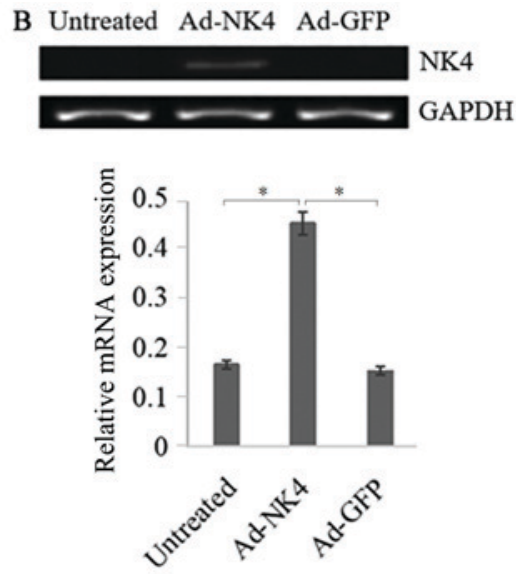

Figure 1. NK4 protein and mRNA expression in RPMI 8226 cells untreated or treated with Ad-GFP or Ad-NK4. (A) NK4 protein expression was detected by western blotting. (B) NK4 mRNA levels were analyzed by reverse transcription-quantitative polymerase chain reaction. GAPDH served as control. Data were obtained from three independent experiments. ${ }^{*} \mathrm{P}<0.05$. Ad, adenovirus; GFP, green fluorescent protein; NK4, a splice variant of hepatocyte growth factor in which the heavy chain consists of the N-terminal domain and the four kringle domains.

Reverse transcription polymerase chain reaction ( $R T-P C R)$ analysis. Total RNA was isolated from untreated, Ad-NK4-transduced and Ad-Control-transduced RPMI 8226 cells using TRIzol reagent (Beyotime Institute of Biotechnology, Haimen, China). mRNA was reverse transcribed to cDNA and the PCR was performed using the Thermoscript RT-PCR System kit (Gibco; Thermo Fisher Scientific, Inc.) according to the manufacturer's protocols. Primers were as follows: NK4 forward 5'-CACGGAAGAGGAGATGAGA-3' and reverse 5'-AGGCCTGGCAAGCTTCATTA-3'; GAPDH forward 5'-AGCCTCAAGATCATCAGC-3' and reverse 5'-GAG TCCTTCCACGATACC-3'. PCR amplification consisted of initial denaturation at $94^{\circ} \mathrm{C}$ for $2 \mathrm{~min}$, then 35 cycles as follows: $15 \mathrm{sec}$ at $94^{\circ} \mathrm{C}$ for denaturation, $30 \mathrm{sec}$ at $58^{\circ} \mathrm{C}$ for annealing and $45 \mathrm{sec}$ at $72^{\circ} \mathrm{C}$ for elongation. Bands separated on $1.5 \%$ agarose gels were stained by ethidium bromide and quantified with an image analyzer using Gel-Pro analyzer 4.0 software (Media Cybernetics, Rockville, MD, USA).

Cell proliferation assay. Untreated, Ad-NK4-transduced and Ad-Control-transduced RPMI 8226 cells were seeded in quadruplicate at a density of $2 \times 10^{3}$ cells/well in 96-well plates and cultured at $37^{\circ} \mathrm{C}$ for 24,48 and $72 \mathrm{~h}$. RPMI 1640 medium without cells was provided as the blank control. Subsequently, $20 \mu 1$ MTT (Sigma-Aldrich; Merck KGaA, Darmstadt, Germany) substrate ( $5 \mathrm{mg} / \mu 1$ in PBS) was added to each well and the plates were incubated at $37^{\circ} \mathrm{C}$ for $4 \mathrm{~h}$. Following a centrifugation at $400 \mathrm{x} \mathrm{g}$ at $4^{\circ} \mathrm{C}$ for $5 \mathrm{~min}$, supernatants were carefully removed and $200 \mu \mathrm{l}$ dimethyl sulfoxide was added to each well. Insoluble crystals were dissolved and a colorimetric analysis was performed at $570 \mathrm{~nm}$. The inhibition rate was calculated as follows: 100\%-[optical density (OD) Ad-NK4 group/OD blank control] $\%$.

Flow cytometry analysis. A total of $48 \mathrm{~h}$ after transduction, $2 \times 10^{5}$ untreated, Ad-NK4-transduced and Ad-Control-transduced RPMI 8226 cells were washed twice with ice-cold PBS and fixed with $70 \%$ ethanol at $4^{\circ} \mathrm{C}$ overnight. Following washing with PBS, cells were incubated in $0.5 \mathrm{ml} \mathrm{PBS}$ containing $50 \mu \mathrm{g} / \mathrm{ml}$ RNase A for $30 \mathrm{~min}$ at $37^{\circ} \mathrm{C}$ and then propidium iodide (PI)

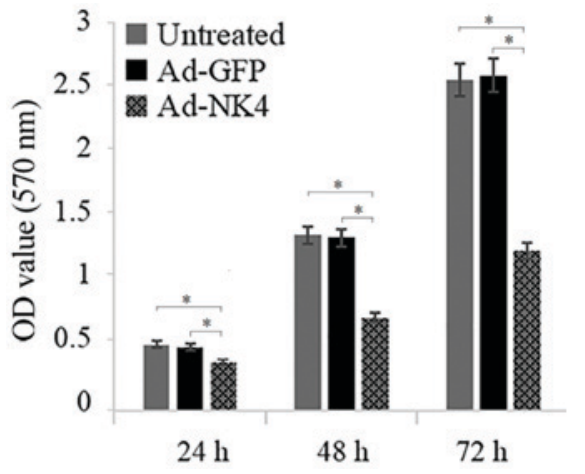

Figure 2. NK4 inhibits RPMI 8226 cell proliferation. Cell proliferation was measured by MTT assay in RPMI 8226 cells untreated, treated with Ad-GFP or Ad-NK4 for 24, 48 and $72 \mathrm{~h}$. Data were obtained from three independent experiments. ${ }^{*} \mathrm{P}<0.05$. Ad, adenovirus; GFP, green fluorescent protein; OD, optical density; NK4, a splice variant of hepatocyte growth factor in which the heavy chain consists of the N-terminal domain and the four kringle domains.

was added to achieve the final concentration of $50 \mu \mathrm{g} / \mathrm{ml}$ and incubated for $30 \mathrm{~min}$ on ice in the dark following the protocols of a propidium iodide staining kit (Sigma-Aldrich; Merck $\mathrm{KGaA}$ ). Samples were subjected to flow cytometry analysis (Coulter Epics XL; Beckman Coulter, Inc., Brea, CA, USA). The percentage of apoptotic cells (sub-G1) and cells in the $G_{0} / G_{1}, S$ and $\mathrm{G}_{2} / \mathrm{M}$ phases were calculated.

Western blot analysis. Following transduction, untreated, Ad-NK4-transduced and Ad-Control-transduced RPMI 8226 cells were collected by centrifugation at $400 \mathrm{x}$ g at $4^{\circ} \mathrm{C}$ for $5 \mathrm{~min}$ and lysed. Protein concentration was determined using a bicinchoninic acid (BCA) Protein Assay kit (Thermo Fisher Scientific, Inc.) following the manufacturer's protocol. Equal amounts of protein (20 $\mu \mathrm{g} /$ lane) were separated on $10 \%$ SDS/PAGE gels and transferred onto polyvinylidene fluoride membranes, which were blocked with TBST containing 5\% skimmed milk at $4^{\circ} \mathrm{C}$ overnight, and then incubated with primary antibodies for cyclin D1 (cat. no. sc-56302), apoptosis regulator Bcl-2 (Bcl-2; cat.no. sc-7382), apoptosis regulator BAX 


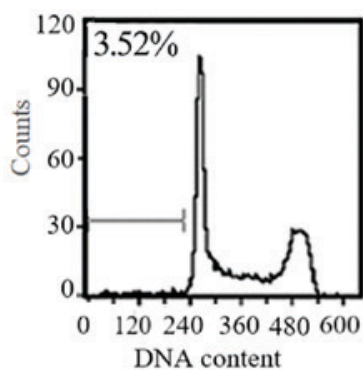

Untreated

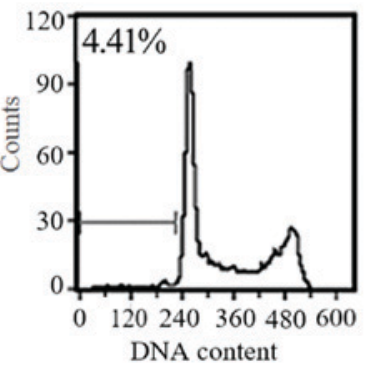

Ad-GFP

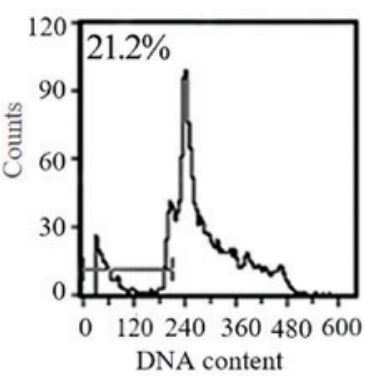

Ad-NK4

Figure 3. Flow cytometry analysis of NK4 induced apoptosis in RPMI 8226 cells. RPMI 8226 cells were untreated, treated with Ad-GFP or Ad-NK4 for 48 h, the cells at sub-G1 phase were analyzed by flow cytometry. The data were obtained from three independent experiments. Ad, adenovirus; GFP, green fluorescent protein; NK4, a splice variant of hepatocyte growth factor in which the heavy chain consists of the N-terminal domain and the four kringle domains.

(Bax; cat. no. sc-20067), cyclin-dependent kinase 4 (CDK4; cat. no. sc-70831), phosphorylated (p)-serine/threonine-protein kinase mTOR (p-mTOR; cat. no. sc-293133), NK4 (cat. no. sc-166724), cyclin-dependent kinase inhibitor 1B (P27; cat. no. sc-53906; all 1:500; Santa Cruz Biotechnology, Inc., Dallas, TX, USA), RAC- $\alpha$ serine/threonine-protein kinase (Akt; cat. no. sc-5298), p-Akt (cat. no. sc-271966; both 1:1,000; Santa Cruz Biotechnology, Inc.), cleaved caspase-3 (cat. no. 9661), cleaved caspase-9 (cat. no. 9505), p-ribosomal protein S6 kinase $\beta$-1 (p70S6K; cat. no. 9208) and p-eukaryotic initiation factor 4E binding protein 1 (cat. no. 9456; all 1:500; Cell Signaling Technology, Inc., Danvers, MA, USA) for $2 \mathrm{~h}$ at room temperature. Following washing with TBST, membranes were incubated with horseradish peroxidase-conjugated secondary antibodies (cat. no. sc-2357; 1:1,500; Santa Cruz Biotechnology, Inc.) for $2 \mathrm{~h}$ at room temperature. Blots were visualized by using the enhanced chemiluminescence reagent kit (Beyotime Institute of Biotechnology) and analyzed by using Quantity One 4.0 software (Bio-Rad Laboratories, Inc., Hercules, CA, USA).

DNA fragmentation assay. Following transduction, untreated, Ad-NK4-transduced and Ad-Control-transduced RPMI 8226 cells were harvested by centrifugation at $1,600 \mathrm{x} \mathrm{g}$ for 5 min at $4^{\circ} \mathrm{C}$ and washed twice using cold PBS. DNA was extracted using the DNA ladder extraction kit (Beyotime Institute of Biotechnology) according to the manufacturer's protocol and separated on $1 \%$ agarose gels. DNA ladders were visualized using a Bio-Rad GelDoc XR System (Bio-Rad Laboratories, Inc.).

Statistical analysis. Data are presented as the mean \pm standard deviation and analyzed by SPSS software for Windows (version 19.0; IBM Corp., Armonk, NY, USA). All experiments were repeated at least three times. The significance of differences between two groups was analyzed by Student's t-test, and the significance of differences among multiple groups was analyzed by analysis of variance followed by post-hoc Tukey's test. $\mathrm{P}<0.05$ was considered to indicate a statistically significant difference.

\section{Results}

NK4 overexpression in RPMI 8226 cells. To confirm adenovirus-mediated overexpression of NK4 in RPMI 8226 cells,

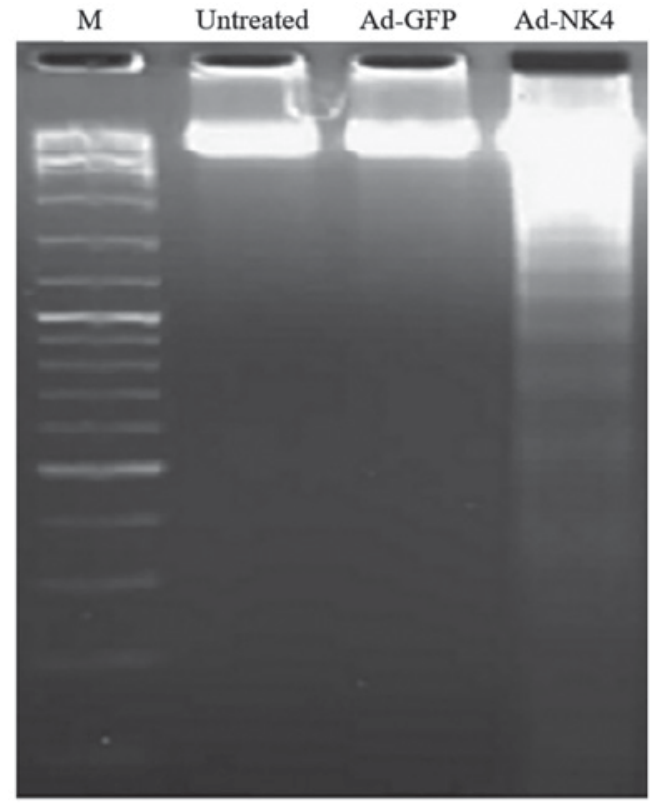

Figure 4. DNA fragmentation assay of NK4 induced apoptosis in RPMI 8226 cells. RPMI 8226 cells were untreated, treated with Ad-GFP or Ad-NK4. DNA ladder was detected by agarose gel electrophoresis. Ad, adenovirus; GFP, green fluorescent protein; M, DNA marker; NK4, a splice variant of hepatocyte growth factor in which the heavy chain consists of the N-terminal domain and the four kringle domains.

RT-qPCR and western blot analysis were conducted. The results presented a significant increase in overexpression of NK4 mediated by AD-NK4 in RPMI 8226 cells compared with the untreated and Ad-GFP RPMI 8226 cells (Fig. 1).

NK4 inhibits proliferation of RPMI 8226 cells. It was investigated whether NK4 decreased the proliferation of RPMI 8226 cells. As presented in Fig. 2, compared with the RPMI 8226/Ad-GFP and the untreated RPMI 8226 group, the proliferation ability of RPMI 8226/Ad-NK4 was inhibited by $33.51 \pm 1.76 \%, 52.79 \pm 1.88 \%$ and $54.24 \pm 2.76 \%$ at 24,48 and $72 \mathrm{~h}$, respectively.

$N K 4$ induces $G_{0} / G_{1}$ arrest and apoptosis in RPMI 8226 cells. To elucidate how NK4 affects cell proliferation, cell cycle and apoptosis were analyzed. The percentage of cells in $\mathrm{G}_{0} / \mathrm{G}_{1}$ phase in RPMI 8226/Ad-NK4 
A Untreated Ad-GFP Ad-NK4
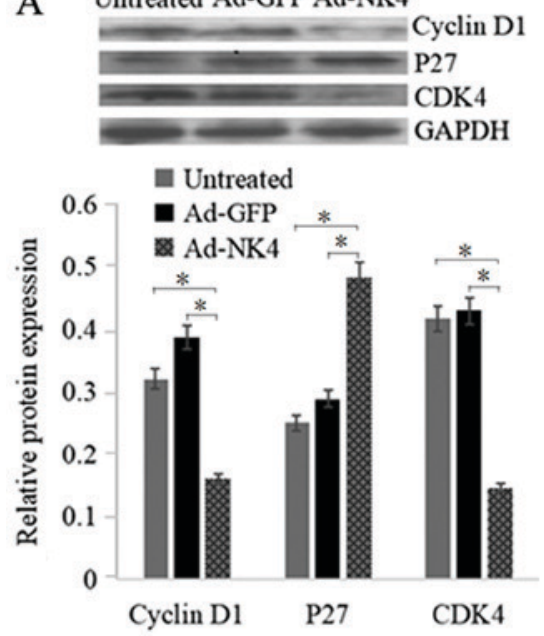

B Untreated Ad-GFP Ad-NK4
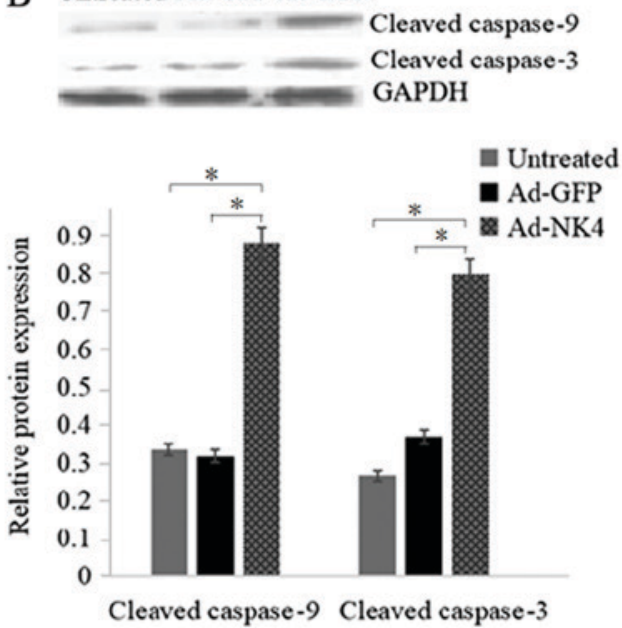

C Untreated Ad-GFP Ad-NK4
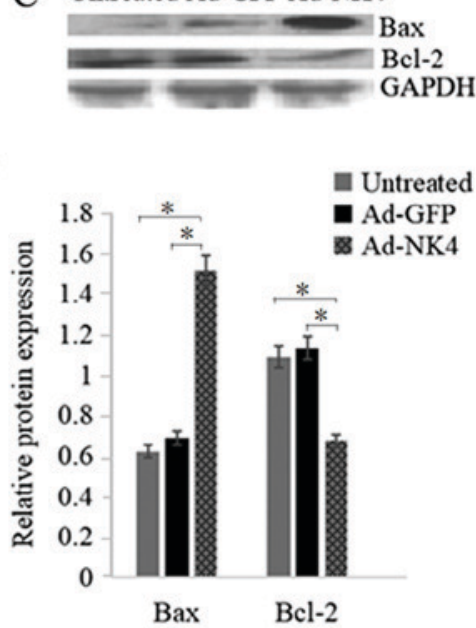

Figure 5. NK4 modulates the expression of cell proliferation and apoptosis-associated proteins. RPMI 8226 cells were untreated, treated with Ad-GFP or Ad-NK4 for $48 \mathrm{~h}$ and protein levels were detected by western blot analysis. (A) Cell cycle-associated proteins, including cyclin D, P27 and CDK4. (B) Apoptosis-associated proteins, including cleaved caspase 3 and 9. (C) Proteins of the Bcl-2 family, including Blc-2 and Bax. GAPDH served as loading control. Data were obtained from three independent experiments. " $\mathrm{P}<0.05$. Ad, adenovirus; GFP, green fluorescent protein; P27, cyclin-dependent kinase inhibitor 1B; CDK4, cyclin-dependent kinase 4; Bcl-2, apoptosis regulator Bcl-2; Bax, apoptosis regulator BAX; NK4, a splice variant of hepatocyte growth factor in which the heavy chain consists of the $\mathrm{N}$-terminal domain and the four kringle domains.

$(53.13 \pm 2.37 \%)$ was increased significantly compared with the RPMI 8226/Ad-GFP $(24.41 \pm 1.76 \%)$ and the untreated RPMI 8226 group $(21.18 \pm 1.98 \%$; data not shown). The percentage of cells in S phase in RPMI 8226/Ad-NK4 $(23.12 \pm 1.65 \%)$ was decreased significantly compared with the RPMI 8226/Ad-GFP $(47.31 \pm 1.87 \%)$ and the untreated RPMI 8226 group $(49.66 \pm 2.07 \%$; data not shown). In order to determine the apoptosis in RPMI 8226 cells, a flow cytometry-based analysis of PI-stained cells and DNA fragmentation electrophoresis was performed. As presented in Fig. 3, the apoptotic rate in RPMI 8226/Ad-NK4 (21.23 $\pm 1.76 \%)$ was increased significantly compared with the RPMI 8226/Ad-GFP $(4.51 \pm 0.29 \%)$ and the untreated RPMI 8226 group $(3.7 \pm 0.32 \%)$. The DNA fragmentation assay demonstrated that DNA isolated from RPMI 8226 cells transduced by Ad-NK4 ran like a ladder on agarose electrophoresis, indicating apoptosis in these cells (Fig. 4).

NK4 regulates cell cycle and apoptosis-associated proteins in RPMI 8226 cells. To further elucidate how NK4 affects cell cycle and apoptosis, western blot analysis was performed. As presented in Fig. 5A, protein levels of CDK4 and cyclin D1 were significantly downregulated in RPMI 8226/Ad-NK4 compared with the RPMI 8226/Ad-GFP and the untreated RPMI 8226. Protein levels of p27 were significantly upregulated in RPMI 8226/Ad-NK4 cells compared with the RPMI 8226/Ad-GFP and untreated RPMI 8226 cells. As presented in Fig. 5B and C, protein levels of Bax, cleaved caspase-9 and cleaved caspase-3 significantly increased, whereas protein levels of Bcl-2 decreased in RPMI 8226/Ad-NK4 cells compared with the RPMI 8226/Ad-GFP and the untreated RPMI 8226 cells.

NK4 regulates the Akt/mTOR signaling pathway in RPMI 8226 cells. To clarify the role of NK4 on the Akt/mTOR signaling pathway and the proliferation and apoptosis of

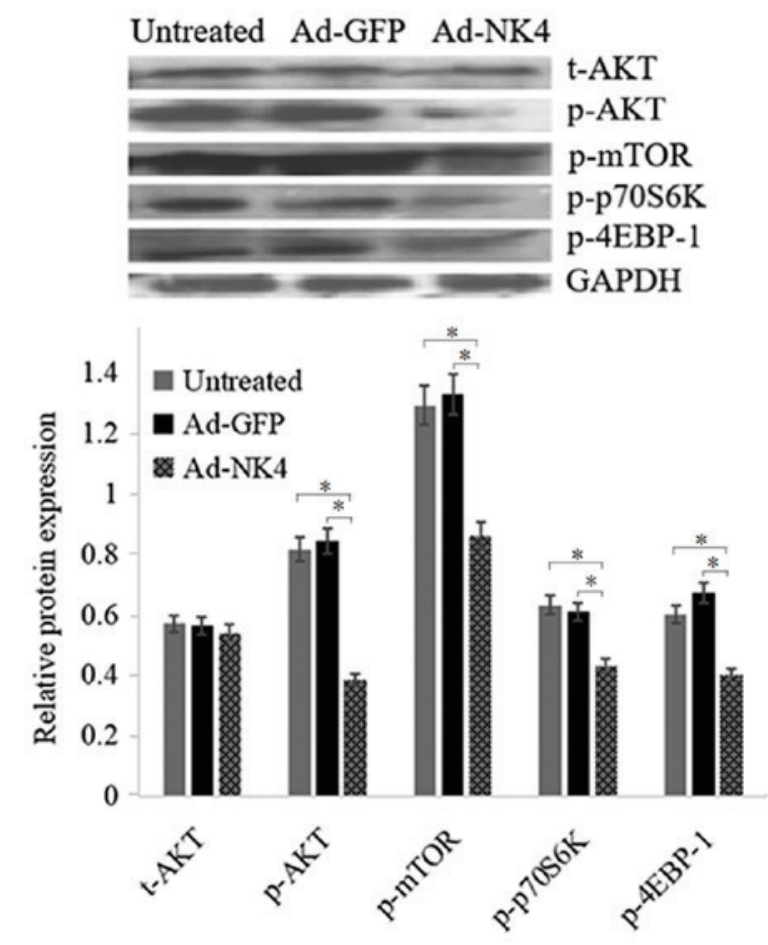

Figure 6. NK4 inhibits the Akt/mTOR signaling pathway. RPMI 8226 cells were untreated, treated with Ad-GFP or Ad-NK4 for $48 \mathrm{~h}$ and protein levels of t-Akt, p-Akt, p-mTOR, p-p70S6K and p-4EBP-1 were detected by western blot analysis. GAPDH served as loading control. Data were obtained from three independent experiments. " $\mathrm{P}<0.05$. Ad, adenovirus; GFP, green fluorescent protein; p, phosphorylated; Akt, RAC- $\alpha$ serine/threonine-protein kinase; mTOR, serine/threonine-protein kinase mTOR; p70S6K, ribosomal protein S6 kinase beta-1; 4E-BP1, eukaryotic initiation factor 4E binding protein 1; t-Akt, total Akt; NK4, a splice variant of hepatocyte growth factor in which the heavy chain consists of the $\mathrm{N}$-terminal domain and the four kringle domains.

RPMI 8226 cells, western blot analysis was performed to validate the status of Akt/mTOR signaling. As presented in Fig. 6, levels of p-AKT, p-mTOR, p-p70S6K and p-4BEP-1 
significantly decreased in RPMI 8226/Ad-NK4 cells compared with the RPMI 8226/Ad-GFP and the untreated RPMI 8226 cells. No significant difference in the expression level of total Akt in RPMI 8226/Ad-NK4 cells compared with the RPMI 8226/Ad-GFP and the untreated RPMI 8226 cells was observed.

\section{Discussion}

Previous studies indicated that NK4 inhibits proliferation and induces apoptosis in several cancer cell lines (11-13). However, antimyeloma activities of NK4 remain to be identified. The current study demonstrated that NK4 possessed antimyeloma effects in vitro. NK4 significantly inhibited the proliferation of human MM RPMI 8226 cells. However, Ad-mediated overexpression of NK4 was not sufficient to completely inhibit the proliferation of MM RPMI 8226 cells, as treated cells still grew at $72 \mathrm{~h}$. It has previously been reported that Ad-NK4 enhances the chemosensitivity of MM RPMI 8226 cells to bortezomib, the first proteasome inhibitor to be used as anti-cancer drug (10). These results suggest that a combined therapy with Ad-NK4 and chemotherapeutic agents may be an effective treatment of MM.

Cell proliferation inhibition is known to be associated with the induction of apoptosis and cell cycle arrest $(14,15)$. Flow cytometry analysis in the present study demonstrated that NK4 induced apoptosis and cell cycle arrest at G0/G1 in RPMI 8226 cells. A DNA fragmentation assay confirmed the induction of apoptosis by NK4. Members of the Bcl-2 family, including anti- and proapoptotic regulators, serve key roles in cell survival and apoptosis (16). It was reported that NK4 increased 5-fluorouracil sensitivity in cholangiocarcinoma cells by modulating the expression of the Bcl-2 family (17). In the present study, the antiapoptotic protein Bcl-2 was downregulated, whereas proapoptotic protein Bax was upregulated by NK4, suggesting that NK4 modulated the expression of Bcl-2 family members in RPMI 8226 cells. Caspase family proteins are executors of apoptosis (18). The present study demonstrated that the expression of cleaved caspase- 3 and caspase-9 significantly increased in NK4-treated RPMI 8226 cells compared with untreated or Ad-GFP-treated cells. In addition, the expression levels of cyclin D1 and CDK4, two cell cycle-promoting proteins, were downregulated whereas the levels of p27, a growth suppressor protein, were upregulated by NK4, suggesting that NK4 induced cell cycle arrest. In conclusion, these results demonstrated that NK4 induced apoptosis and arrested cell cycle in RPMI 8226 cells.

Using recombinant Ad containing NK4 cDNA, Du et al (19) reported that NK4 inhibits growth and induces apoptosis in MM cells. These effects may be associated with the inhibition of the activation of c-Met, extracellular signal-regulated kinase1/2, signal transducer and activator of transcription factor 3 and Akt (19). Akt/mTOR signaling serves a crucial role in the regulation of cell proliferation, cell cycle and apoptosis (20). A previous study revealed that knockdown of c-Met enhances the sensitivity to bortezomib in human MM U266 cells by inhibiting Akt/mTOR activation Therefore, in the present study, the mechanism by which NK4 exhibits anti-MM activity was examined and the results demonstrated that NK4 may inhibit Akt/mTOR signaling in MM cells.
In conclusion, it was demonstrated that NK4 inhibited cell proliferation by inducing apoptosis and cell cycle arrest in RPMI 8226 cells. The apoptotic effect of NK4 may be associated with decreased Bcl-2 level, increased Bax and cleaved caspase 3 and 9 levels and inhibiting Akt/mTOR signaling in RPMI 8226 cells. These findings suggest that NK4 may be a potential agent for the treatment of MM. Further investigations are necessary to explore the detailed mechanism of the underlying apoptotic effect of NK4 on human MM cells both in vitro and in vivo.

\section{Acknowledgements}

Not applicable.

\section{Funding}

The present study was supported by the National Natural Science Foundation of China (grant no. 81500167) and Fujian Provincial Clinical Key Subject Construction Project.

\section{Availability of data and materials}

The datasets used and/or analyzed during the current study are available from the corresponding author on reasonable request.

\section{Authors' contributions}

WQ and SX designed the study. WQ, HL and QY performed the experiments. All authors read and approved the manuscript.

\section{Ethics approval and consent to participate}

Not applicable.

\section{Patient consent for publication}

Not applicable.

\section{Competing interests}

The authors declare that they have no competing interests.

\section{References}

1. Bergsagel PL, Mateos MV, Gutierrez NC, Rajkumar SV and San Miguel JF: Improving overall survival and overcoming adverse prognosis in the treatment of cytogenetically high-risk multiple myeloma. Blood 121: 884-892, 2013.

2. Zaman S, Shentu S, Yang J, He J, Orlowski RZ, Stellrecht CM and Gandhi V: Targeting the pro-survival protein MET with tivantinib (ARQ 197) inhibits growth of multiple myeloma cells. Neoplasia 17: 289-300, 2015.

3. Borset M, Lien E, Espevik T, Helseth E, Waage A and Sundan A: Concomitant expression of hepatocyte growth factor/scatter factor and the receptor c-MET in human myeloma cell lines. J Biol Chem 271: 24655-24661, 1996.

4. Mahtouk K, Tjin EP, Spaargaren M and Pals ST: The $\mathrm{HGF} / \mathrm{MET}$ pathway as target for the treatment of multiple myeloma and B-cell lymphomas. Biochim Biophys Acta 1806: 208-219, 2010.

5. Que W, Chen J, Chuang M and Jiang D: Knockdown of c-Met enhances sensitivity to bortezomib in human multiple myeloma U266 cells via inhibiting Akt/mTOR activity. APMIS 120: 195-203, 2012. 
6. Que W and Chen J: Knockdown of c-Met inhibits cell proliferation and invasion and increases chemosensitivity to doxorubicin in human multiple myeloma U266 cells in vitro. Mol Med Rep 4: 343-349, 2011

7. Ferrucci A, Moschetta M, Frassanito MA, Berardi S, Catacchio I, Ria R, Racanelli V, Caivano A, Solimando AG, Vergara D, et al: A HGF/cMET autocrine loop is operative in multiple myeloma bone marrow endothelial cells and may represent a novel therapeutic target. Clin Cancer Res 20: 5796-5807, 2014.

8. Mizuno S and Nakamura T: HGF-MET cascade, a key target for inhibiting cancer metastasis: The impact of NK4 discovery on cancer biology and therapeutics. Int J Mol Sci 14: 888-919, 2013.

9. Que W and Chen J: Gateway technical supported construction of a recombinant adenovirus vector pAd-NK4. Chinese Pharmacol Bulletin 27: 467-472, 2011

10. Que W and Chen J: Ad-NK4 enhances the chemosensitivity of human multiple myeloma RPMI 8226 cells to bortezomib. Zhongguo Shi Yan Xue Ye Xue Za Zhi 24: 1079-1085, 2016 (In Chinese).

11. Deng XB, Xiao L, Wu Y, Jin F, Mossman B, Testa JR and Xiao GH: Inhibition of mesothelioma cancer stem-like cells with adenovirus-mediated NK4 gene therapy. Int J Cancer 137: 481-490, 2015

12. Ramanujum R, Lin YL, Liu JK and He S: Regulatory expression of MMP-8/MMP-9 and inhibition of proliferation, migration and invasion in human lung cancer A549 cells in the presence of HGF variants. Kaohsiung J Med Sci 29: 530-539, 2013.

13. Sun YP, Zhang BL, Duan JW, Wu HH, Wang BQ, Yu ZP Yang WJ, Shan YF, Zhou MT and Zhang QY: Effect of NK4 transduction in bone marrow-derived mesenchymal stem cells on biological characteristics of pancreatic cancer cells. Int J Mol Sci 15: 3729-3745, 2014.
14. Han L, Zhang Y, Liu S, Zhao Q, Liang X, Ma Z, Gupta PK, Zhao M and Wang A: Autophagy flux inhibition, G2/M cell cycle arrest and apoptosis induction by ubenimex in glioma cell lines. Oncotarget 8: 107730-107743, 2017.

15. Lima KG, Krause GC, da Silva EFG, Xavier LL, Martins LAM, Alice LM, da Luz LB, Gassen RB, Filippi-Chiela EC, Haute GV, et al: Octyl gallate reduces ATP levels and Ki67 expression leading HepG2 cells to cell cycle arrest and mitochondria-mediated apoptosis. Toxicol In Vitro 48: $11-25,2017$.

16. Kale J, Osterlund EJ and Andrews DW: BCL-2 family proteins: Changing partners in the dance towards death. Cell Death Differ 25: 65-80, 2018

17. Ge X, Wang Y, Li Q, Yu H, Ji G and Miao L: NK4 regulates 5-fluorouracil sensitivity in cholangiocarcinoma cells by modulating the intrinsic apoptosis pathway. Oncol Rep 30: 448-454, 2013.

18. Zhang T, Ji D, Wang P, Liang D, Jin L, Shi H, Liu X, Meng Q, $\mathrm{Yu} \mathrm{R}$ and Gao S: The atypical protein kinase RIOK3 contributes to glioma cell proliferation/survival, migration/invasion and the AKT/mTOR signaling pathway. Cancer Lett 415: 151-163, 2018.

19. Du W, Hattori Y, Yamada T, Matsumoto K, Nakamura T, Sagawa M, Otsuki T, Niikura T, Nukiwa T and Ikeda Y: NK4, an antagonist of hepatocyte growth factor (HGF), inhibits growth of multiple myeloma cells: Molecular targeting of angiogenic growth factor. Blood 109: 3042-3049, 2007.

20. Lv XY, Ma L, Chen JF, Yu R, Li Y, Yan ZJ, Cheng Y and Ma Q: Knockdown of DUXAP10 inhibits proliferation and promotes apoptosis in bladder cancer cells via PI3K/Akt/mTOR signaling pathway. Int J Oncol 52: 288-294, 2018. 